\title{
DEUX NÉMATODES NOUVEAUX D'UN SINGE HURLEUR DE SURINAME
}

\author{
Par P.-H. VAN THIEL
}

Parmi les vers trouvés par le docteur C. Bonne à Suriname, danș l'intestin d'un singe hurleur (Alouata $=$ Mycetes seniculus L.), vers qu'il m'a envoyés à déterminer, j'ai trouvé deux nématodes nouveaux, dont la description suit :

\section{Syphacia bonnei n. sp.}

(Fig. 1-5)

Femelle. - Longueur $8 \mathrm{~mm}$., 5 ; largeur maxima $480 \mu$. Corps courbé, souvent légèrement,-en forme de faucille (fig. 1,1 ) et couvert par une cuticule striée transversalement, les stries étant espacées de $11 \mu, 5$ au milieu du corps, mais à une distance moindre dans la région céphalique et manquant dans la région caudale, qui est grêle et allongée.

A gauche et à droite de la région buccale, il y a une expansion cuticulaire (fig. 1, 2), qui est, contrairement à ce qui a lieu chez la plupart des oxyures, très large et élégante. A $84 \mu$ de l'extrémité céphalique, ces expansions se prolongent de chaque côté en deux crêtes longitudinales semi-cylindriques très étroites (1).

Bouche entourée de trois lèvres assez larges ; en avant de l'expansion cuticulaire, la pulpe du corps envoie dans la partie antérieure du corps six prolongements, trois grands et trois un peu plus petits, de façon à ce que les grands se trouvent sous les lèvres et les petits en face des incisions entre les lèvres. Cavité buccale courte; œesophage musculaire, de $1 \mathrm{~mm}$., 90 de longueur, séparé du bulbe oesophagique (diamètre de $144 \mu$ ) par un col court. L'intestin, un peu plus large en avant qu'en arrière, se rend avec quelques courbures vers la queue, où il se termine à l'anus, à $1 \mathrm{~mm} ., 48$ en avant de la pointe caudale. La dernière partie de l'intestin constitue le rectum, avec deux glandes rectales à sa base.

Chez deux individus immatures la partie postérieure de l'intestin,

(1) Martini dessine deux crêtes semblables chez le Passalurus ambiguus. Il est probable que Seurat a décrit sous le nom de " deux ailes latérales étroites », la même structure chez le Syphacia obvelata.

Annales de Parasitologie, T. III, No 2. - Avril 1925, p. 171-180. 

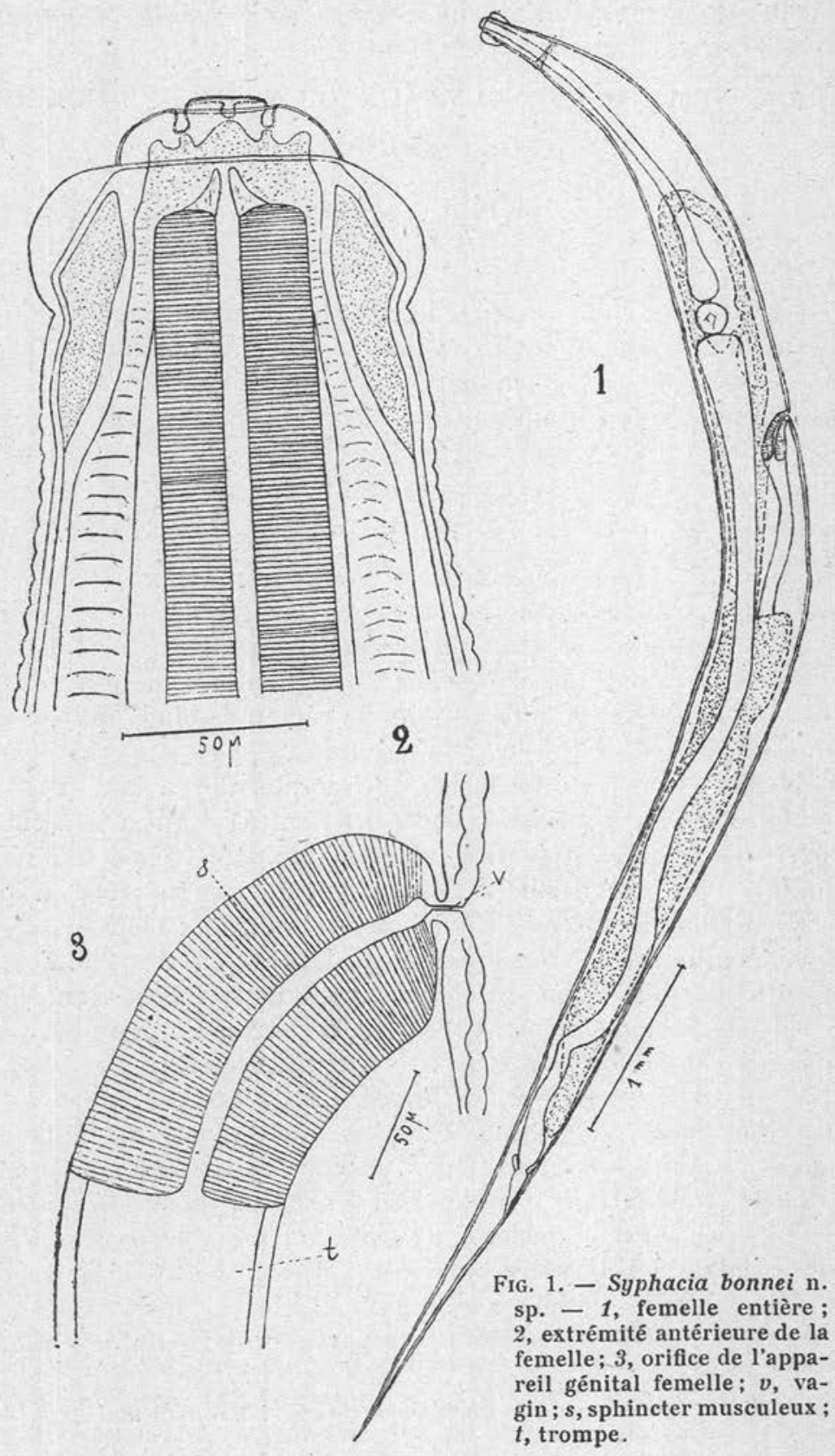
en avant du rectum, était fortement pliée, comme le montre la figure 4, structure qui m'est inconnue.

L'utérus bicorne occupe le milieu du corps et est rempli d'œufs,
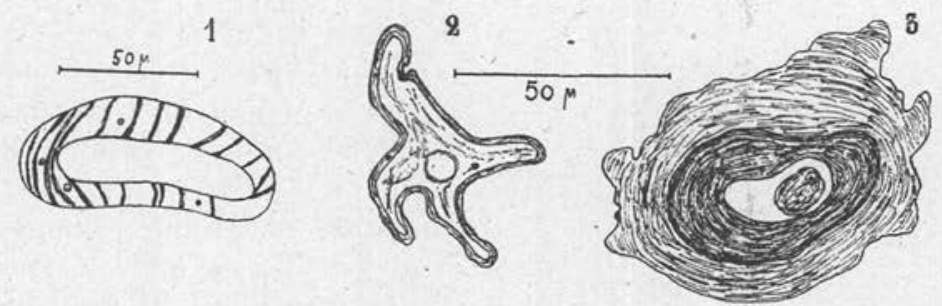

Fıg. 2. - Syphacia bonnei n. sp. - 1, sphincter de l'ovéjecteur, coupe longitudinale ; 2 , coupe transversale de la trompe; 3 , coupe transversale de la "basitrompe ».

ce qui rend les ovaires invisibles sur la plus grande partie de leur parcours.-Les deux branches de l'utérus sont divergentes; la

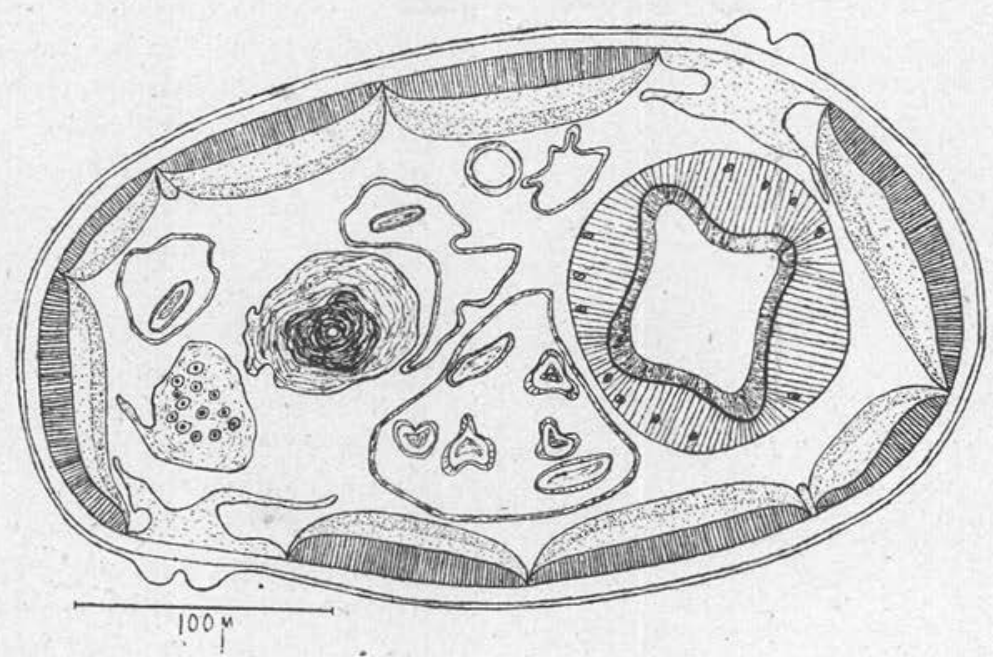

Fig. 3. - Syphacia bonnei n. sp. - Coupe transversale de la femelle.

branche antérieure (largeur maxima : $106 \mu$ ) s'étend jusqu'à $828 \mu$ en avant du bulbe œsophagien ; la postérieure (largeur maxima : $148 \mu$ ). jusqu'à $288 \mu$ en avant de l'anus. La vulve (fig. 1, 3), non saillante, située à $2 \mathrm{~mm}$., 75 de l'extrémité céphalique, se trouve donc au tiers antérieur du corps. L'ovéjecteur (1) est composé d'un

(1) Nomenclature de M. Seurat. 
très court vagin, sans vestibule et d'un sphincter musculeux très fort (fig. 2, 1). La trompe est très allongée, à parois fortement pliées,

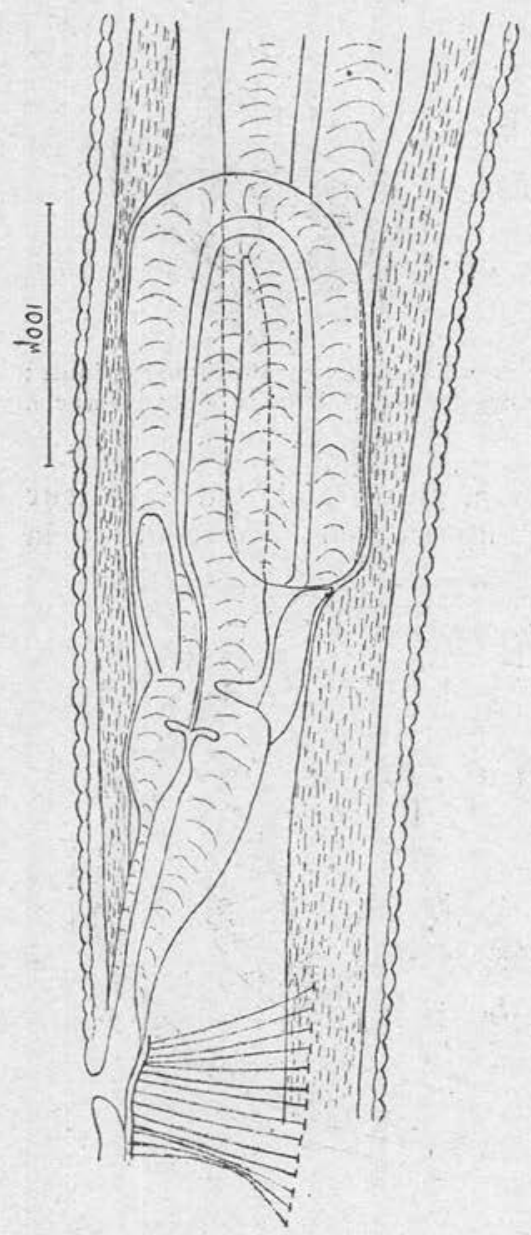

FIG. 4. - Spyhacia bonnei n. sp. Femelle immature, partie postérieure au niveau du rectum. comme le montre la figure 2,2 . Il m'a été impossible d'en mesurer la longueur exacte, car elle est recouverte par l'utérus. Pourtant je suis persuadé que cet organe doit être considéré comme long, ayant pu l'étudier chez un ver immature de $6 \mathrm{~mm}$. de longueur. Chez ce spécimen, l'ovéjecteur mesurait $88 \mu$ et la trompe $390 \mu$; chez l'individu adulte, ces mesures étant 198 et au moins $360 \mu$. La dernière partie de la trompe, près de l'endroit où elle se continue avec l'utérus, est plus grande que le reste et sa paroi est fortement épaissie (fig. 2, 3) ; la partie centrale de la paroi se colore en rouge foncé avec l'éosine. Je propose pour cette portion de la trompe, dont la structure n'est pas connue jusqu'ici, le nom de " basitrompe " (fig. 3).

Les œufs, aplatis sur une face, mesurent $41 \mu, 8$ de longueur sur $28 \mu, 8$ de largeur; la coque est striée par de nombreux pores qui se distinguent surtout sur une coupe transversale. Alors on voit en même temps, que la coque n'est pas partout aussi épaisse et qu'il y a trois parties assez épaisses et trois autres très minces (fig. 5). Les embryons, au moment de la ponte, sont probablement peu développés.

Le pore excréteur est invisible. L'anneau nerveux est situé à $300 \mu$ de l'extrémité céphalique.

D'après les caractènes de la femelle, ce nématode pourrait être classé soit parmi les Oxyuridæ, soit parmi les Heterakidæ; toutefois il appartient à la première de ces familles, surtout parce que les 
vers de cette famille ont une musculature du type méromyaire, caractère qui sert à distinguer les Oxyuridæ des Heterakidæ (Railliet, Travassos).

Les mâles étant absents, la détermination du genre présente des difficultés, car la systématique est surtout basée sur l'appareil génital de ceux-ci (Railliet et Henry). Pour les raisons suivantes, je range l'oxyure nouveau dans le genre Syphacia Seurat, 1916: trompe impaire, très allongée, non différenciée en un organe d'emmagasinement des œufs; vulve en rapport par un court vagin avec un ovéjecteur cuticulaire remarquable par l'épaisseur de son asșise musculaire ; enfin concordance avec les autres caractères donnés
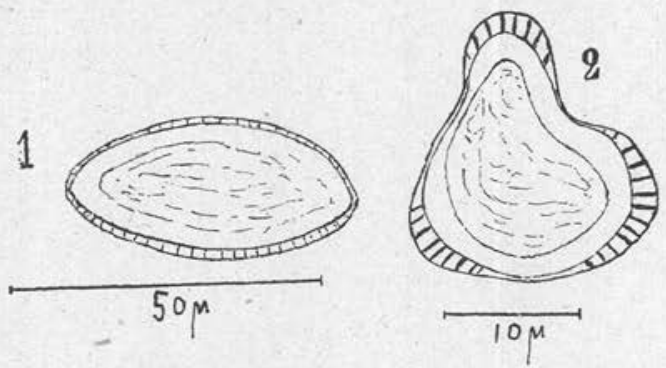

Fı́. 5. - Syphacia bonnei n. sp. - 1, œuf dans l'utérus ; 2, œuf dans une coupe de l'utérus.

par Seurat. Le ver décrit n'est pas le Trypanoxyuris trypanuris Ververs, que Ortlepp a décrit il y a quelque temps chez un singe à Suriname.

L'espèce décrite est-elle la même que l'Oxyuris minuta, recueilli chez Mycetes seniculus et Ateles paniscus au Brésil et décrit par Schneider en 1866 ? Les deux espèces correspondent par les caractères suivants : dimensions de la femelle, longueur de l'œsophage, situation de la vulve, utérus bicorne, coque de l'œuf ponctuée. O. minuta est caractérisée en outre par la présence de quatre papilles distinctes dans la région céphalique, la fréquence relative des mâles ; en outre la forme des crêtes longitudinales est probablement autre que chez notre nouvelle espèce. Schneider mentionne seulement la présence de cette crête et ne rapporte que " die Gestalt des Quenschnitts der Seitenlinie $n$ est autre que chez l'Enterobius vermicularis.

De ces diverses considérations, il résulte que l'oxyure trouvé par Bonne, est une espèce nouvelle et je la lui dédie sous le nom de Syphacia bonnei n. sp. 
Squamanema bonnei n. g., n. sp.

(fig. 6-9)

Femelle immature. - Ver polymaire. Longueur totale : $20 \mathrm{~mm}$., épaisseur maxima : $270 \mu$. Corps filiforme, bouclé en spirale, terminé par une queue obtuse. Stries cuticulaires écartées de $6 \mu, 3$, au milieu du corps, absentes dans la région céphalique et

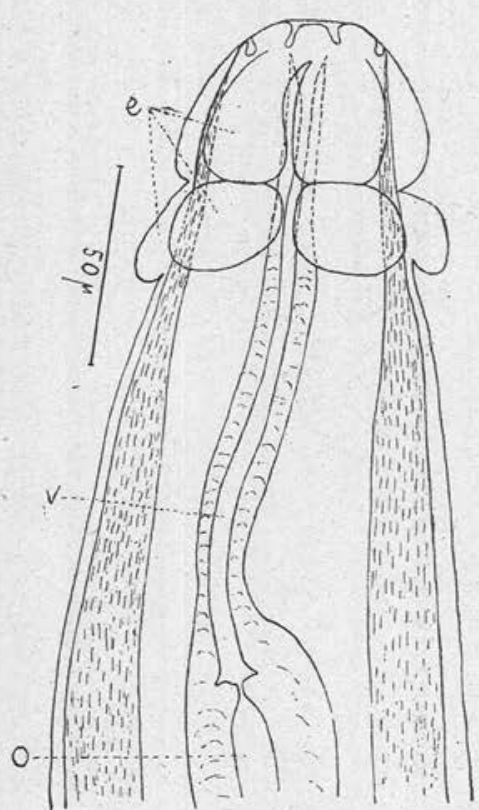

Fig. 6. - Squamanema bonnei n. g., n. sp. - Extrémité antérieure de la femelle; $e$, écussons céphaliques ; $v$, vestibulum oris ; $o$, œsophage.

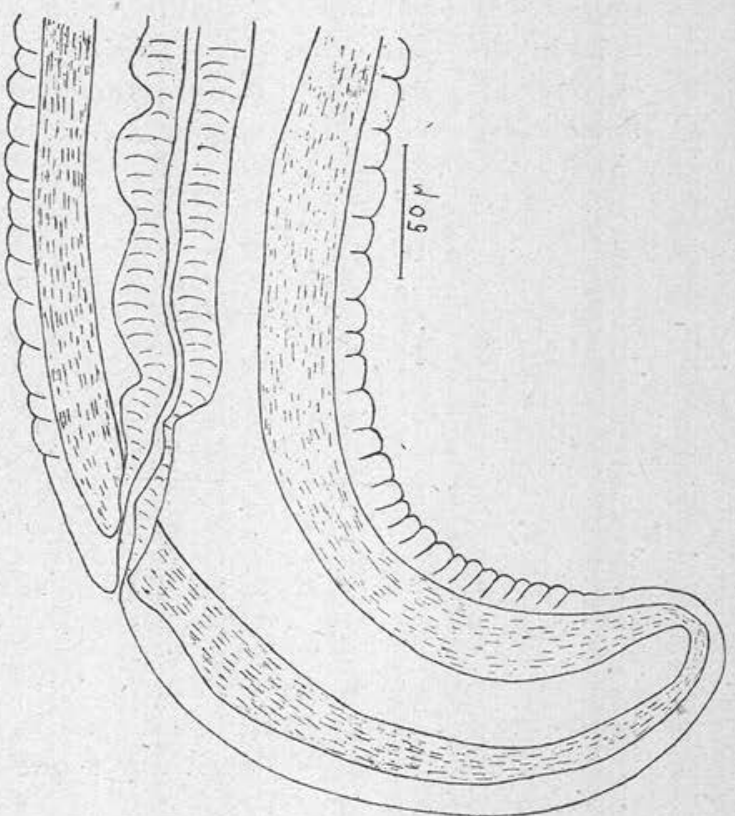

Fig. 7. - Squamanema bonnei n. g., n. sp. - Extrémité postérieure de la femelle.

cans la partie postérieure de la queue. Dans la région céphalique la cuticule forme dorsalement et ventralement quatre écussons, qui sont en partie en contact les uns avec les autres (fig. 6).

La cuticule est très fortement épaissie d'un seul côté du corps et atteint, au milieu du corps même, une épaisseur de $41 \mu, 8$, mais elle devient plus mince vers la région céphalique et vers la queue. Cet épaississement présente vers son milieu une incision longitudinale assez marquée (fig. 9, 2).

La bouche triangulaire, encadrée de six lèvres, dont trois sont un peu plus petites que les autres, donne accès dans un vestibule 
de $180 \mu$ de longueur et de $16 \mu, 8$ de largeur. L'œsophage, plus large que le vestibule et long de $0 \mathrm{~mm}$., 9 , se recourbe comme l'intestin dans le reste du corps. Anus à $223 \mu$ de la pointe caudale.

Il existe deux papilles cervicales latérales légèrement saillantes

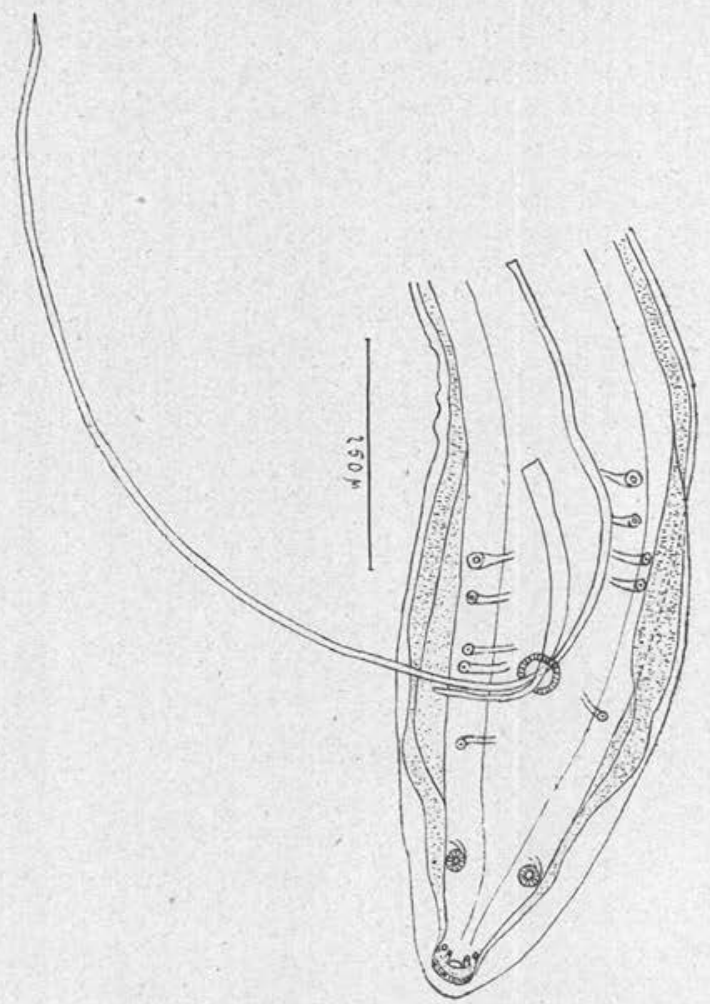

Fig. 8. - Squamanema bonnei n. g., n. sp.

Extrémité postérieure du mâle vue par la face ventrale.

situées en avant de l'anneau nerveux, à $252 \mu$ de l'extrémité céphalique.

La vulve légèrement saillante est située au tiers postérieur du corps ; l'ovéjecteur et la trompe sont invisibles. Des deux utérus, l'antérieur se coude à $1 \mathrm{~mm}$., du commencement de l'intestin et se dirige en arrière; le postérieur se dirige vers la queue, s'y replie et se termine par l'ovaire à $1 \mathrm{~mm} ., 9$ de la pointe caudale (fig. 7 ). Les utérus, les oviductes et les ovaires sont fortement contournés et se replient de temps en temps dans une autre direction. OEufs invisibles. 
Male. - Plus petit que la femelle, filiforme, bouclé en spirale comme cette dernièrc. Longueur totale: $13 \mathrm{~mm}$., épaisseur maxima: $224 \mu$. Epaisseur cuticulaire comme chez la femelle, mais plus étroite, de $26 \mu$ au plus.

Le testicule présente une forte courbure, mais ne forme pas de replis comme le font de temps en temps les utérus.

La queue, fortement tordue, est ornée de deux ailes un peu iné-

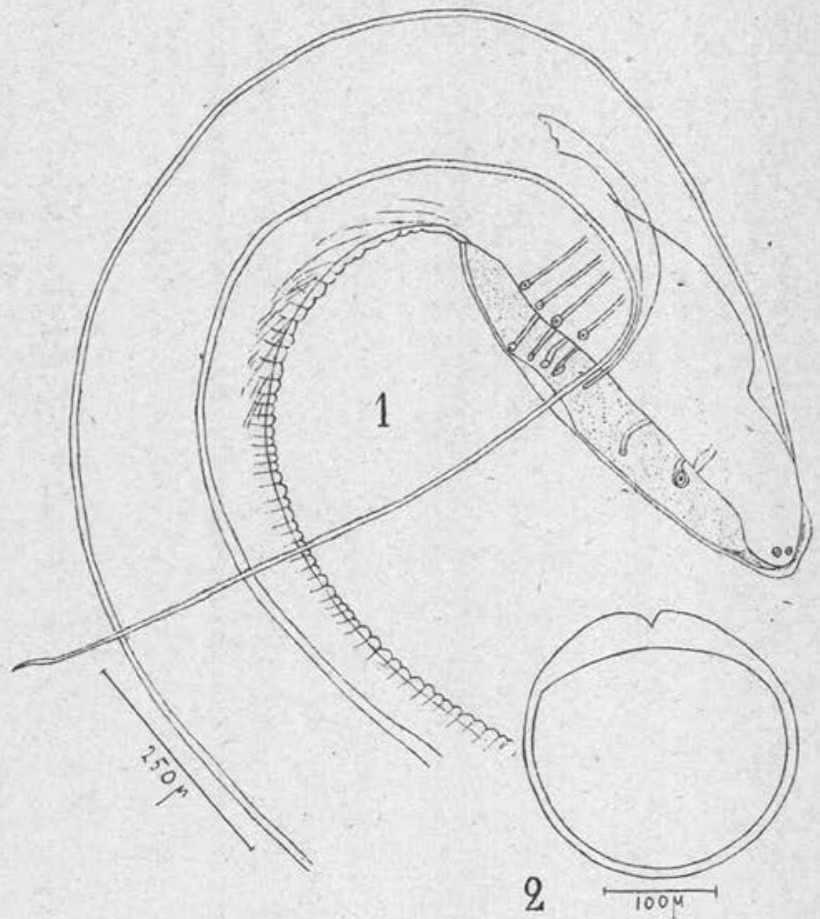

Fıg. 9. - Squamanema bonnei n. g., n. sp. - 1, extrémité postérieure du mâle vue de profil ; 2 , coupe transversale de la femelle au niveau du tiers antérieur.

gales dont la droite est la plus longue et mesure $612 \mu$. Ces deux ailes caudales s'incurvent vers la face ventrale du corps et arrivent même au contact l'une de l'autre. Cloaque limité par un anneau un peu musculeux. Spicules très inégaux, le droit court, le gauche filiforme, moins large que l'autre et présentant une pointe fine à son extrémité.

Pores caudaux subterminaux, situés à $24 \mu$ de la pointe caudale. La disposition des papilles génitales est asymétrique : à gauche et 
à droite quatre papilles préanales pédonculées et deux papilles postanales pédonculées, dont la terminale est la plus grande et présente des muscles comme une ventouse (fig. 8 et 9,1 ).

Ce nématode appartient à la famille des Spiruridæ Diesing et se rapproche du genre Gongylonema Molin, quand on le prend dans le sens de Molin, cité par Stossich. Pourtant, en étudiant la description que Seurat donne du même genre, on trouve qu'il y a bien des différences, résidant dans la structure des ailes marginales, dans la présence de quatre lèvres avec trois paires de papilles, dont deux portent une dent interne dans la forme de l'œsophage et dans le genre de vie à l'intérieur de la muqueuse de l'œsophage ou de l'estomac des mammifères.

Pour ces diverses raisons, je place cette espèce dans un nouveau genre: le genre Squamanema, qui diffère du genre Gongylonema surtout par la structure de la bouche et par le nombre réduit des écussons céphaliques et œsophagiens. Je dédie ce parasite à celui qui l'a trouvé et lui donne le nom de Squamanema bonnei, n. g., n. sp.

Je ne veux pas terminer cette étude sans avoir attiré l'attention sur le Gongylonema filiforme, que Molin a déerit chéz un singe hurleur. D'après la description et les figures de ce ver, cet exemplaire est un vrai Gongylonema et diffère de celui que je décris ici.

\section{RÉsumé}

Dans la présente note, je signale deux nématodes nouveaux d'un singe hurleur (Mycetes seniculus L.) ; ce sont: Syphacia bonnei et Squamanema bonnei.

\section{BibLIOGRAPHIE}

Martinı (E.). - Ueber Subcuticula und Seitenfelder einiger Nematoden IV. Ztschr.

f. wiss. Zool., XCIII, 1909, p. 535.

Molin (R.). - Notizie elmintologiche. Atti dell' I. R. Inst. Veneto, II, 1856-1857, p. 220. OrtLePp (R.-J.). - On a collection of Helminths from Dutch Guiana. Journ. of Helminthology, II, 1924, p. 15.

Rallliet (A.) et Henry (A.). - Sur les Oxyuridés. C. $R$. Soc. Biol., LXViII, 1916, p. 113.

Schneiden (A.). - Monographie der Nematoden. Berlin, 1866.

Seurat (L -G.). - Sur les Oxyures des Mammifères. C. R. Soc. Biol., LXVIII, 1916 , p. 64. 
Seurat (L.-G.). - Sur les Gongylonèmes du Nord-Africain. C. R. Soc. Biol., LXVIII, 1916, p. 717.

Sтоssich (M.). - Filarie e Spiroptere. Lavoro monografico. Bull. d. Soc. Adriat. d. Sz. nat. in Trieste, XVIII, 1898.

Travassos (L.). - Contribution à l'étude de la faune helminthologique du Brésil. Memorias do Inst. Osw. Cruz., XII, 1920, p. 41.

Vevers (G.-M.). - Some new and little known Helminths from British Guiana. Journ. of Helminthology, I, 1923, p. 35.

Institut de médecine tropicale et Laboraloire de pathologie comparée el de parasitologie à Leyde. 